\title{
A NEW DIMENSION FUNCTION
}

\author{
J. M. AARTS
}

ABSTRACT. A new inductive dimension function, Hind, is defined for hereditarily normal spaces. The countable and locally finite sum theorems for Hind are proved for hereditarily normal spaces. It is shown that Hind = Ind on the class of totally normal spaces.

1. Introduction. The finite sum theorem for the strong inductive dimension Ind does not hold in general for compact Hausdorff spaces. The counterexample is due to Lokucievskii [4], [5, Example 16-6]. It is an open question whether the finite sum theorem holds for hereditarily normal spaces. The positive results which are due to Dowker are as follows. In the class of totally normal spaces the countable sum theorem, the open subset theorem and the subset theorem for Ind hold [1], [5, \$11]. There is also a locally finite sum theorem for Ind in totally normal spaces [3], [5, Lemma 25-3]. The proofs of these results are strongly interrelated.

In this note we introduce a new inductive dimension function, Hind, for hereditarily normal spaces for which the sum theorems can be proved without relying on an open subset theorem. These theorems are valid in the class of hereditarily normal spaces and the proofs are straightforward. It is to be noted that the (open) subset theorem for Ind as well as Hind does not hold in the class of hereditarily normal spaces. The counterexample which is due to Filippov [2] will be discussed in $\$ 2$.

The dimension function Hind agrees with Ind on the class of totally normal spaces. Also, Hind $X=$ Ind $X$ for every hereditarily normal space $X$ with Hind $X \leq 1$. So by the defining of Hind we have singled out the property which is crucial for the sum theorems of the inductive dimension.

It is an open question whether Hind and Ind agree on the class of hereditarily normal spaces. This question is related to that mentioned above. A negative answer to the former question provides a negative answer to the latter.

2. Definition of Hind. The huge inductive dimension Hind is defined for every hereditarily normal space $X$ as follows:

Received by the editors May 1, 1974 .

AMS (MOS) subject classifications (1970). Primary 54F 45.

Key words and phrases. Inductive dimension, sum theorems. 
(1) Hind $X=-1$ if and only if $X=\varnothing$;

(2) For each integer $n \geq 0$, Hind $X \leq n$ provided that for each pair of closed subsets $F$ and $G$ with $\operatorname{Hind}(F \cap G) \leq n-1$ there is a pair of closed subsets $K$ and $L$ such that $F \backslash G \subset K \backslash L, G \backslash F \subset L \backslash K, K \cup L=X$ and Hind $(K \cap L) \leq n-1$.

Hind $X=n, n=0,1,2, \cdots, \infty$, are defined as usual.

Observe that in (2) the set $K \cap L$ separates $F \backslash G$ and $G \backslash F$ in $X$. This proves the "only if' -part of the following proposition.

Proposition 1. For each integer $n \geq 0$, Hind $X \leq n$ if and only if for each pair of closed subsets $F$ and $G$ with Hind $(F \cap G) \leq n-1$ there is a closed subset $S$ such that $F \backslash G$ and $G \backslash F$ are separated by $S$ and Hind $S \leq n-1$.

Proof. The "if"'-part is proved as follows. Let $F$ and $G$ be closed subsets with $\operatorname{Hind}(F \cap G) \leq n-1$ and let $S$ be a closed subset which separates $F \backslash G$ and $G \backslash F$ and which has Hind $\leq n-1$. Then $X \backslash S=U \cup V$ where $U$ and $V$ are disjoint open sets containing $F \backslash G$ and $G \backslash F$ respectively. Then $K=U \cup S$ and $L=V \cup S$ satisfy the conditions required in the definition of Hind $X \leq n$.

Corollary 1. Let $n=-1$ or 0 . Hind $X=n$ if and only if Ind $X=n$ for every hereditarily normal space $X$.

The easy inductive proofs of the following propositions are omitted.

Proposition 2. Ind $\leq$ Hind on the class of hereditarily normal spaces.

Proposition 3. Let $C$ be a closed subset of a hereditarily normal space $X$. Then Hind $C \leq$ Hind $X$.

From Corollary 1 and Proposition 2 it follows that Hind $X=$ Ind $X$ for every hereditarily normal space $X$ with $\operatorname{Hind} X \leq 1$.

Example 1. Filippov [2] has given an example of a hereditarily normal and zero-dimensional space $X$ which contains subspaces $X_{n}, n=1,2, \cdots$, with Ind $X_{n}=n$. The construction of the example is based on the assumption of the existence of a Suslin tree. In view of Corollary 1 and Proposition 2 the same example shows the failure of the subset theorem for Hind in the class of hereditarily normal spaces. It is to be observed that the open subset theorem and the subset theorem for Ind are equivalent in the class of hereditarily normal spaces [1], [5, $\$ 11-2]$. So there is also no open subset theorem for Hind in the class of hereditarily normal spaces. 
3. The sum theorems. The proofs of the sum theorems are based on the following lemmas.

Lemma 1. Let $F$ and $G$ be closed subsets of a hereditarily normal space $X$. Then in $X$ there exist closed neighborhoods $U$ and $V$ of $F \backslash G$ and $G \backslash F$, respectively, such that $U \cap V=F \cap G$.

Proof. $F \backslash G$ and $G \backslash F$ are disjoint closed subsets of the normal subspace $X_{1}=X \backslash(F \cap G)$. So in $X_{1}$ there are disjoint closed neighborhoods $U_{1}$ and $V_{1}$ of $F \backslash G$ and $G \backslash F$ respectively. As $X_{1}$ is open, $U_{1}$ and $V_{1}$ are also neighborhoods in $X$. Now let $U=F \cup U_{1}$ and $V=G \cup V_{1}$. As $\operatorname{cl}_{X} U_{1}$ $\subset U_{1} \cup(F \cap G), U$ is closed in $X$. Similarly $V$ is closed.

$U \cap V=F \cap G$, because $U_{1} \cap G \subset F \cap G$ and also $V_{1} \cap F \subset F \cap G$.

Lemma 2. Let $F$ and $G$ be closed subsets of a hereditarily normal space $X$ and let $Y$ be a subset of $X$ such that $Z=F \cup G \cup Y$ is closed in $X$. Suppose Hind $Y \leq n$ and $\operatorname{Hind}(F \cap G \cap Y) \leq n-1$. Then there exist closed subsets $K$ and $L$ of $X$ and a closed subset $S$ of $Y$ such that

(1) $F \backslash G \subset K \backslash L$ and $G \backslash F \subset L \backslash K$;

(2) $K \cup L=Z$;

(3) $K \cap L=(F \cap G) \cup S$; and

(4) Hind $S \leq n-1$.

Proof. By Lemma 1 in $X$ there are closed neighborhoods $U$ and $V$ of $F \backslash G$ and $G \backslash F$, respectively, such that $U \cap V=F \cap G$. By virtue of Proposition 1 there is a partition $\{Q, R, S\}$ of $Y$, where $Q$ and $R$ are open and $S$ is closed in $Y$, such that $(U \backslash V) \cap Y \subset Q,(V \backslash U) \cap Y \subset R$ and Hind $S \leq n-1$. Now let $K=F \cup Q \cup S$ and $L=G \cup R \cup S$. First observe that $V \backslash U$ is a neighborhood of $G \backslash F$. As $(Q \cup S) \cap(V \backslash U)=\varnothing, \mathrm{cl}_{X}(Q \cup S) \cap(G \backslash F)=\varnothing$. Hence $\mathrm{cl}_{X}(Q \cup S) \subset K$, because $Z$ is closed. It follows that $K$ is closed in $X$. Similarly, $L$ is closed. We also have $(Q \cup S) \cap G \subset F \cap G$ and, similarly, $(R \cup S) \cap F \subset G \cap F$. So $K \cap L=(F \cap G) \cup S$. Clearly $S \cap(F \cup G) \subset F \cap G$. It follows that $K \backslash L \supset F \backslash(K \cap L)=F \backslash G$. Now it is easily seen that (1)-(4) are satisfied.

Remark 1. If in Lemma 2 we suppose that Ind $Y \leq n$ and $F \cap G \cap Y=\varnothing$ (instead of Hind $Y \leq n$ and $\operatorname{Hind}(F \cap G \cap Y) \leq n-1)$, in the same way it can be shown that there exist closed subsets $K$ and $L$ of $X$ and a closed subset $S$ of $Y$ satisfying (1), (2), (3) and Ind $S \leq n-1$.

We now prove the countable sum theorem.

Theorem 1. Let $\left\{X_{i} \mid i=1,2, \ldots\right\}$ be a countable closed cover of the 
hereditarily normal space $X$, such that Hind $X_{i} \leq n, i=1,2, \cdots$. Then Hind $X \leq n$.

Proof. The proof is by induction on $n$. The theorem is obvious for $n=-1$. Suppose it has been proved for $n-1$. Let $K_{0}$ and $L_{0}$ be closed subsets of $X$ such that for $D_{0}=K_{0} \cap L_{0}$ we have Hind $D_{0} \leq n-1$. Inductively on $i$ we shall define closed subsets $D_{i}, K_{i}$ and $L_{i}$ of $X, i=1,2, \cdots$, such that for each $i$ :

(1) $K_{i-1} \backslash L_{i-1} \subset$ int $_{X} K_{i} \backslash L_{i}$ and $L_{i-1} \backslash K_{i-1} \subset$ int $_{X} L_{i} \backslash K_{i}$;

(2) $X_{i} \subset K_{i} \cup L_{i}$;

(3) $D_{i}=K_{i} \cap L_{i}$;

(4) $D_{i-1} \subset D_{i}$; and

(5) Hind $D_{i} \leq n-1$.

Suppose $D_{j}, K_{j}$ and $L_{j}$ have already been defined for $j=0,1, \cdots, i-1$ satisfying (1)-(5). In view of (3) and Lemma 1 there are closed neighborhoods $U$ and $V$ of $K_{i-1} \backslash L_{i-1}$ and $L_{i-1} \backslash K_{i-1}$, respectively, such that $U \cap V=$ $D_{i-1}$.

By applying Lemma 2 (with $F, G$ and $Y$ replaced by $U, V$ and $X_{i}$ respectively) we get closed sets $K_{i}, L_{i}$ and $S$ such that, if we define $D_{i}=$ $K_{i} \cap L_{i}$, then (1)-(3) above are satisfied, $D_{i}=D_{i-1} \cup S$ and Hind $S \leq n-1$. Clearly (4) is satisfied and (5) follows from the induction hypothesis.

Finally let $U=\bigcup\left\{K_{i} \backslash L_{i} \mid i=1,2, \cdots\right\}, V=\bigcup\left\{L_{i} \backslash K_{i} \mid i=1,2, \cdots\right\}$ and $D=\bigcup\left\{D_{i} \mid i=1,2, \cdots\right\}$. Then $\{U, V, D\}$ is a partition of $X$ by virtue of (1)-(4). $U$ and $V$ are open in view of (1). Hence $D$ is closed. By the induction hypothesis and (5) we get Hind $D \leq n-1$. By Proposition 1 we have Hind $X \leq n$, since $K_{0} \backslash L_{0} \subset U$ and $L_{0} \backslash K_{0} \subset V$ in view of (1).

The locally finite sum theorem is as follows.

Theorem 2. Let $\left\{X_{\alpha} \mid \alpha \in A\right\}$ be a locally finite closed cover of a hereditarily normal space $X$, such that Hind $X_{\alpha} \leq n$ for each $\alpha \in A$. Then Hind $X$ $\leq n$.

Proof. The proof is by induction on $n$. The theorem is obvious for $n=$ -1 . Suppose it has been proved for $n-1$. Let $K_{0}$ and $L_{0}$ be subsets of $X$ such that for $D_{0}=K_{0} \cap L_{0}$ we have Hind $D_{0} \leq n-1$. Suppose $\left\{X_{a} \mid \alpha \in A\right\}$ is well ordered as $\left\{X_{a} \mid 1 \leq \alpha<\tau\right\}$. Let $Z_{\alpha}=\bigcup\left\{X_{\beta} \mid 1 \leq \beta \leq \alpha\right\}$ for $1 \leq \alpha<\tau$. Let $Z_{0}=\varnothing$. By transfinite induction on $\alpha$ we shall define closed subsets $K_{\alpha}, L_{\alpha}$ and $D_{\alpha}$ of $X$ such that for all $\alpha, \beta, \gamma<\tau$ :

(1) $K_{\beta} \backslash L_{\beta} \subset K_{\gamma} \backslash L_{\gamma}$ and $L_{\beta} \backslash K_{\beta} \subset L_{\gamma} \backslash K_{\gamma}$ if $\beta<\gamma$; 
(2) $K_{\alpha} \cup L_{a}=Z_{\alpha} \cup\left(K_{0} \cup L_{0}\right)$;

(3) $D_{\alpha}=K_{a} \cap L_{\alpha}$;

(4) $D_{\beta} \subset D_{\gamma}$ for $\beta<\gamma$; and

(5) Hind $D_{\alpha} \leq n-1$.

Suppose $D_{\beta}, K_{\beta}$ and $L_{\beta}$ have been defined for all $\beta<\alpha$ satisfying (1)-(5) for ordinals less than $\alpha$.

Let $F=\bigcup\left\{K_{\beta} \mid \beta<\alpha\right\}, G=\bigcup\left\{L_{\beta} \mid \beta<\alpha\right\}$ and $T=\bigcup\left\{D_{\beta} \mid \beta<\alpha\right\}$. In view of (1) and (3) we have $F \cap G=T$. Observe that $T=\left(K_{0} \cap L_{0}\right) \cup$ $\left\{D_{\beta} \cap X_{\beta} \mid 1 \leq \beta<\alpha\right\}$, because, if $x \in T \backslash\left(K_{0} \cap L_{0}\right)$, then for the minimal $\gamma<\alpha$ with $x \in X_{y}$ we also have $x \in D_{y}$ in view of (1) and (3). Note that $\left\{D_{\beta} \cap X_{\beta} \mid \beta<\alpha\right\}$ is a locally finite closed collection. In view of Proposition 3 and the induction hypothesis, Hind $T \leq n-1$. It also follows that $T$ is closed. Similarly $F$ and $G$ can be shown to be closed.

By applying Lemma 2 (with $Y$ replaced by $X_{a}$ ) we get closed sets $K_{a}$, $L_{a}$ and $S$ such that, if we define $D_{a}=K_{\alpha} \cap L_{a}$, then (1)-(3) above are satisfied for ordinals less than or equal to $\alpha, D_{\alpha}=T \cup S$ and Hind $S \leq n-1$. Trivially (4) holds for ordinals not exceeding $\alpha$ and (5) follows from Theorem 1.

Finally let $F^{*}=\bigcup\left\{K_{a} \mid \alpha<\tau\right\}, G^{*}=\bigcup\left\{L_{\alpha} \mid \alpha<\tau\right\}$ and $T^{*}=\bigcup\left\{D_{a} \mid \alpha<\tau\right\}$. In the same way as has been done for $F, G$ and $T$, it is shown that $F^{*}, G^{*}$ and $T^{*}$ are closed, that $T^{*}=F^{*} \cap G^{*}$ and that Hind $T^{*} \leq n-1$. The construction ensures that $K_{0} \backslash L_{0} \subset F^{*} \backslash G^{*}$ and $L_{0} \backslash K_{0} \subset G^{*} \backslash F^{*}$ in view of (1). Hence Hind $X \leq n$.

The following corollary will be useful in establishing the equality of Ind and Hind in the class of totally normal spaces. This result is also in [1].

Corollary 2. Let the hereditarily normal space $X$ be the disjoint union of a closed subspace $X_{1}$ and an open subspace $X_{2}$. If Ind $X_{1} \leq n$ and Ind $X_{2} \leq n$, then Ind $X \leq n$.

Proof. The proof is by induction and the case $n=-1$ is obvious. Assume the theorem has been proved for $n-1$. Let $K_{0}$ and $L_{0}$ be disjoint closed subsets of $X$. Let $D_{1}, K_{1}$ and $L_{1}$ be constructed as in the proof of Theorem 2 in such a way that Ind $D_{1} \leq n-1$. It is to be noted that $D_{1} \cap$ $X_{2}=\varnothing$. In view of Remark 1 by Lemma 2 (with $F, G$ and $Y$ replaced by $K_{1}$, $L_{1}$ and $X_{2}$ respectively) we get closed subsets $K$ and $L$ of $X$ and a closed subset $S$ of $X_{2}$ such that $K_{0} \subset K \backslash L, L_{0} \subset L \backslash K, K \cup L=X, K \cap L=D_{1} \cup S$ and Ind $S \leq n-1$. By the induction hypothesis Ind $(K \cap L) \leq n-1$. It follows that Ind $X \leq n$. 
4. Results for totally normal spaces. Recall that a normal space $X$ is totally normal if each open subset $U$ of $X$ admits a cover $\mathcal{O}$ such that each $V \epsilon$ $\mathcal{O}$ is a cozero-subset in $X$ and $\mathcal{O}$ is locally finite in $U$. As a cozero-subset of $X$ is an $F_{\sigma}$-subset of $X$, by Proposition 3 and Theorems 1 and 2 we have that Hind $U \leq$ Hind $X$ whenever $U$ is an open subset of a totally normal space $X$.

Thus the following proposition has been proved.

Proposition 4. Let $X$ be a totally normal space and let $U$ be an open subset of $X$. Then Hind $U \leq$ Hind $X$.

We shall now show that Ind and Hind coincide on the class of totally normal spaces.

Theorem 3. Ind = Hind on the class of totally normal spaces.

Proof. In view of Proposition 2 we need only prove that Hind $\leq$ Ind on the class of totally normal spaces. By induction on $n$ we shall prove the following statement.

$E(n)$ : If Ind $X \leq n$, then Hind $X \leq n$ for every totally normal space $X$.

We assume $E(n)$ has been proved for $-1,0, \cdots, n-1$ (cf. Corollary 1). Let $F$ and $G$ be closed subsets of $X$ such that $\operatorname{Hind}(F \cap G) \leq n-1$. Define $Y=X \backslash(F \cap G)$. In view of the open subset theorem for Ind on the class of totally normal spaces $[5, \S 11-5]$ (see also below) Ind $Y \leq n$. By Lemma 2 and Remark 1 there exist closed subsets $K$ and $L$ of $X$ and a closed subset $S$ of $Y$ such that

(1) $F \backslash G \subset K \backslash L$ and $G \backslash F \subset L \backslash K$;

(2) $K \cup L=X$;

(3) $K \cap L=(F \cap G) \cup S$;

(4) Ind $S \leq n-1$.

By Corollary 2 and Proposition 3 we have Ind $(K \cap L) \leq n-1$. By virtue of the induction hypothesis Hind $(K \cap L) \leq n-1$, whence Hind $X \leq n$.

Remark on the open subset theorem for Ind. A proof of the open subset theorem for Ind, which is used in the proof of Theorem 3, can be based on the results in $\$ 3$ and the induction hypothesis. For completeness sake we shall indicate such a proof. Combined with the results of $\$ 3$, this will give a new proof of the sum theorems for Ind.

Assuming $E(n-1)$ we first prove

$A(n)$ : Let $\left\{X_{\alpha} \mid \alpha \in A\right\}$ be a locally finite closed collection of totally normal space $X$ such that $\left\{\right.$ int $\left._{X} X_{a} \mid \alpha \in A\right\}$ is a cover of $X$ and Ind $X_{a} \leq n$ for each $\alpha \in A$. Then Ind $X \leq n$. 
Proof of $A(n)$. (int, $\mathrm{cl}$ and $B$ denote the interior, closure and boundary operator in $X$.) Let $\left\{F_{a} \mid \alpha \in A\right\}$ be a closed cover of $X$ such that $F_{a} \subset$ int $X_{a}$. Suppose $F$ is closed in $X$ and $V$ is an open neighborhood of $F$. Choose open subsets $W_{a}$ such that Ind $B\left(W_{\alpha}\right) \leq n-1$ and $F_{a} \cap F \subset W_{a} C$ cl $W_{a} \subset V \cap$ int $X_{a}$. By $E(n-1)$ and Theorem 2 we get Hind $\bigcup\left\{B\left(W_{\alpha}\right) \mid a \in A\right\}$ $\leq n-1$. Let $W=\bigcup\left\{W_{a} \mid \alpha \in A\right\}$. Then $F \subset W \subset V$ and Hind $B(W) \leq n-1$, because $B(W) \subset \bigcup\left\{B\left(W_{a}\right) \mid a \in A\right\}$ in view of the local finiteness of the last collection. By Proposition 3 and $E(n-1)$ it follows that Ind $B(W) \leq n-1$, whence Ind $X \leq n$.

In a standard fashion from $A(n)$ one can deduce

$O(n)$ : If $U$ is an open subset of a totally normal space $X$ with Ind $X \leq n$, then Ind $U \leq n$.

\section{REFERENCES}

1. C. H. Dowker, Inductive dimension of completely normal spaces, Quart. J. Math. Oxford Ser. (2) 4 (1953), 267-281. MR $16 \# 157$.

2. V. V. Filippov, On the dimension of normal spaces, Soviet Math. Dokl. 14 (1973), 547-550.

3. N. Kimura, On a sum theorem in dimension theory, Proc. Japan Acad. 43 (1967), 98-102. MR 35 \#7311.

4. O. V. Lokucievskii, On the dimension of bicompacta, Dokl. Akad. Nauk SSSR 67 (1949), 217-219. (Russian) MR 11 \#46.

5. K. Nagami, Dimension theory, Pure and Appl. Math., Vol. 37, Academic Press, New York, 1970. MR 42 \#6799.

DEPARTMENT OF MATHEMATICS, DELFT INSTITUTE OF TECHNOLOGY, DELFT, THE NETHERLANDS 